\title{
Submaximal factorizations of a Coxeter element in complex reflection groups
}

\author{
Vivien Ripoll \\ LaCIM, UQÀM, Montréal, QC, Canada
}

\begin{abstract}
When $W$ is a finite reflection group, the noncrossing partition lattice $N C(W)$ of type $W$ is a very rich combinatorial object, extending the notion of noncrossing partitions of an $n$-gon. A formula (for which the only known proofs are case-by-case) expresses the number of multichains of a given length in $N C(W)$ as a generalized Fuß-Catalan number, depending on the invariant degrees of $W$. We describe how to understand some specifications of this formula in a case-free way, using an interpretation of the chains of $N C(W)$ as fibers of a "Lyashko-Looijenga covering". This covering is constructed from the geometry of the discriminant hypersurface of $W$. We deduce new enumeration formulas for certain factorizations of a Coxeter element of $W$.

Résumé. Lorsque $W$ est un groupe de réflexion fini, le treillis $N C(W)$ des partitions non-croisées de type $W$ est un objet combinatoire très riche, qui généralise la notion de partitions non-croisées d'un $n$-gone. Une formule (seulement prouvée au cas par cas à l'heure actuelle) exprime le nombre de chaînes de longueur donnée dans $N C(W)$ sous la forme d'un nombre de Fuß-Catalan généralisé, qui dépend des degrés invariants de $W$. Nous décrivons une stratégie visant à comprendre certaines spécifications de cette formule de manière uniforme, en utilisant une interprétation des chaînes de $N C(W)$ comme fibres d'un "revêtement de Lyashko-Looijenga". Ce revêtement est construit à partir de la géométrie de l'hypersurface du discriminant de $W$. Nous en déduisons de nouvelles formules de comptage pour certaines factorisations d'un élément de Coxeter de $W$.
\end{abstract}

Keywords: complex reflection groups, generalized noncrossing partition lattice, generalized Fuss-Catalan numbers, factorizations of a Coxeter element

\section{Introduction}

Let $W$ be a well-generated irreducible complex reflection group ${ }^{(i)}$ We define a partial order $\preccurlyeq$ on $W$, related to the reflection length of the elements in $W$ (see Def. 3). The noncrossing partition lattice of type $W$, denoted $\mathrm{NC}(W)$, is a particular interval for this order. It is an algebraic generalization of the lattice of noncrossing partitions of an $n$-gon, and it has many important combinatorial properties. One of the most amazing ones is the following:

Proposition 1 ("Chapoton's formula") Let W be an irreducible, well-generated complex reflection group, of rank $n$. Then, for any $p \in \mathbb{N}$, the number of multichains $w_{1} \preccurlyeq \ldots \preccurlyeq w_{p}$ in the poset $\mathrm{NC}(W)$ is equal to

$$
\mathrm{Cat}^{(N)}(W)=\prod_{i=1}^{n} \frac{d_{i}+p h}{d_{i}}
$$

(i) The precise definitions will be given in Sect 2

1365-8050 @ 2011 Discrete Mathematics and Theoretical Computer Science (DMTCS), Nancy, France 
where $d_{1} \leq \cdots \leq d_{n}=h$ are the invariant degrees of $W$ (defined in Sec. 2.1).

The numbers Cat ${ }^{(p)}(W)$ are called Fuß-Catalan numbers of type $W$. They also appear in other combinatorial objects related to the group $W$, e.g. in the context of cluster algebras of finite type.

In the real case, this formula was first observed by Chapoton in [Cha05, Prop. 9]. The proof is caseby-case (using the classification of finite Coxeter groups), and it mainly uses results by Athanasiadis and Reiner [Rei97, AR04]. The remaining complex cases are checked by Bessis in [Bes07] using results of [BC06]. There is still no case-free proof of this formula, even for the simplest case where $p=1$ which gives the cardinality of $\mathrm{NC}(W)$ as the generalized Catalan number $\prod_{i=1}^{n}\left(d_{i}+h\right) / d_{i}$.

This very simple formula naturally motivates the search for a uniform proof that could shed light on the mysterious relation between the combinatorics of $\mathrm{NC}(W)$ and the invariant theory of $W$.

Multichains in $\mathrm{NC}(W)$ are directly related to certain block factorizations of a Coxeter element $c$ of $W$ (see Def. 4). In fact, Chapoton's formula can be reformulated in terms of these factorizations.

In [Bes07], Bessis discovered that some instances of the formula (namely, the number of maximal factorizations of $c$ ) can be explained through the geometry of a map, called the Lyashko-Looijenga covering LL, constructed from the geometry of the discriminant of $W$.

The object of this paper is to explain how, by studying this map in more detail, we can obtain further enumerative results, giving formulas for the number of submaximal factorizations of $c$.

Theorem 2 (cf. Thm. 8 and Cor. 11) Let c be a Coxeter element of $W$ and $\Lambda$ a conjugacy class of elements of reflection length 2 in $\mathrm{NC}(W)$. Then:

(a) the number of block factorizations of c, constituting of $n-2$ reflections and one element of length 2 and of conjugacy class $\Lambda$, is

$$
\left|\operatorname{FACT}_{n-1}^{\Lambda}(c)\right|=\frac{(n-1) ! h^{n-1}}{|W|} \operatorname{deg} D_{\Lambda},
$$

where $D_{\Lambda}$ is a homogeneous polynomial attached to $\Lambda$, determined by the geometry of the discriminant hypersurface of $W$ (see Sec. 4);

(b) the total number of block factorizations of c in $n-1$ factors (or submaximal factorizations) is

$$
\left|\operatorname{FACT}_{n-1}(c)\right|=\frac{(n-1) ! h^{n-1}}{|W|}\left(\frac{(n-1)(n-2)}{2} h+\sum_{i=1}^{n-1} d_{i}\right) .
$$

While the first point is new, the second one is not. In fact, it is a direct consequence of Chapoton's formula. The main interest here is that the proof is geometric and almos (ii) case-free. The structure of the proof is as follows:

1. we use new geometric properties of the morphism LL to prove formula (a);

2. we find a uniform way to compute $\sum_{\Lambda} \operatorname{deg} D_{\Lambda}$, using an algebraic study of the Jacobian of LL;

3. we deduce formula (b) since $\left|\operatorname{FACT}_{n-1}(c)\right|=\sum_{\Lambda}\left|\operatorname{FACT}_{n-1}^{\Lambda}(c)\right|$.

(ii) We have to rely on some structural properties of LL, proved in [Bes07] case-by-case. 
Thus, even if the method used here does not seem directly generalizable to factorizations with fewer blocks, it is a new interesting avenue towards a geometric case-free explanation of Chapoton's formula.

Outline. In Section 2 we give some background and notations about complex reflection groups and the noncrossing partition lattice, and we define the block factorizations of a Coxeter element. Section 3 is devoted to the construction and properties of the Lyashko-Looijenga covering of type $W$, and in particular its relation with factorizations. In Section 4 we give the precise formulas for submaximal factorizations, and explain the steps of the proof.

All the details of the constructions and proofs can be found in $[\overline{\text { Rip10c }} \mid$ and $[\overline{R i p 10 b}]$ (see also [Rip10a] $)$.

\section{The noncrossing partition lattice and block factorizations of a Coxeter element}

\subsection{Complex reflection groups}

First we recall some notations and definitions about complex reflection groups.

For $V$ a finite dimensional complex vector space, we call a reflection (iii) ${ }^{\text {of }} \mathrm{GL}(V)$ an automorphism $r$ of $V$ of finite order and such that the invariant space $\operatorname{Ker}(r-1)$ is a hyperplane of $V$. We call a complex reflection group a finite subgroup of $\mathrm{GL}(V)$ generated by reflections.

A simple way to construct such a group is to take a finite real reflection group (or, equivalently, a finite Coxeter group together with its natural geometric realization) and to complexify it. There are of course many other examples that cannot be seen in a real space. A complete classification of irreducible complex reflection groups has been given by Shephard-Todd in [ST54] : it consists in an infinite series with three parameters and 34 exceptional groups of small ranks. For more details on these groups, we refer to the book [LT09].

We denote by $W$ a subgroup of $\mathrm{GL}(V)$ which is a complex reflection group. Note that for real reflection groups the results of this paper are already interesting (and, most of them, new).

From now on we suppose that $W$ is irreducible of rank $n^{\text {(iv) }}$ If $\left(v_{1}, \ldots, v_{n}\right)$ denotes a basis of $V$, $W$ acts naturally on the polynomial algebra $\mathbb{C}[V]=\mathbb{C}\left[v_{1}, \ldots, v_{n}\right]$. Chevalley-Shephard-Todd's theorem implies that the invariant algebra $\mathbb{C}[V]^{W}$ is again a polynomial algebra, and it can be generated by $n$ algebraically independent homogeneous polynomials $f_{1}, \ldots, f_{n}$ (called the fundamental invariants). The degrees $d_{1}, \ldots, d_{n}$ of these invariants do not depend on the choices for the $f_{i}$ 's (if we require $d_{1} \leq \cdots \leq$ $d_{n}$ ) and they are called the invariant degrees of $W$. As for finite Coxeter groups, we will denote by $h$ the highest degree $d_{n}$ (Coxeter number).

We will also require that $W$ is a well-generated (irreducible) complex reflection group, i.e., it can be generated by $n$ reflections $(\mathrm{v})$ Then there exist in $W$ so-called Coxeter elements, which generalize the usual notion of a Coxeter element in finite Coxeter groups : a Coxeter element $c$ of $W$ is a $e^{2 i \pi / h}$-regular element (in the sense of Springer's regularity), i.e., it is such that there exists $v \in V$ outside the reflecting hyperplanes such that $c v=e^{2 i \pi / h} v$.

(iii) This is called pseudo-reflection by certain authors.

(iv) That is, the linear action on $V$ is irreducible, and the dimension of $V$ is $n$.

(v) This is, of course, always verified in the real case. 


\subsection{The noncrossing partition lattice of type $W$}

Denote by $\mathcal{R}$ the set of all reflections in $W$. For $w$ in $W$, let $\ell(w)$ denote the minimal length of $w$ as a word on the alphabet $\mathcal{R}$. This is called the reflection length or absolute length (vi)

Definition 3 We denote by $\preccurlyeq$ the absolute order on $W$, that is:

$$
u \preccurlyeq v \quad \text { if and only if } \quad \ell(u)+\ell\left(u^{-1} v\right)=\ell(v) .
$$

If $c$ is a Coxeter element of $W$, the noncrossing partition lattice of $(W, c)$ is:

$$
\mathrm{NC}(W, c)=\{w \in W \mid w \preccurlyeq c\} .
$$

It is easy to see that the structure of $\operatorname{NC}(W, c)$ does not depend ${ }^{\text {(vii) }}$ on the choice of the Coxeter element $c$; thus we will just write $\mathrm{NC}(W)$ for short. In the prototypal case of type $A$, where $W=\mathfrak{S}_{n}, \mathcal{R}$ is the set of all transpositions and $c$ is an $n$-cycle; then $\mathrm{NC}(W)$ is isomorphic to the poset of noncrossing partitions of an $n$-gon, as introduced by Kreweras in [Kre72]. In general, the noncrossing partition lattice of type $W$ has a very rich combinatorial structure, we refer to [Arm09, Ch. 1] or [Rip10a, Chap. 0].

\subsection{Multichains in $\mathrm{NC}(W)$ and block factorizations of a Coxeter element}

As described in the introduction, Chapoton's formula expresses the number of multichains in $\mathrm{NC}(W)$. Here we prefer to work with block factorizations of a Coxeter element, which are directly related to multichains.

Definition 4 For a Coxeter element $c,\left(w_{1}, \ldots, w_{p}\right)$ is a block factorization (viii) of $c$ if:

- $\forall i, w_{i} \in W-\{1\}$

- $w_{1} \ldots w_{p}=c$;

- $\ell\left(w_{1}\right)+\cdots+\ell\left(w_{p}\right)=\ell(c)$.

We denote by $\operatorname{FACT}(c)\left(\right.$ resp. FACT $_{p}(c)$ ), the set of block factorizations of c (resp. factorizations in $p$ factors).

Note that the length of $c$ equals the rank of $W$ (denoted by $n$ ), so any block factorization of $c$ determines a composition of the integer $n$, by taking the distribution of the lengths of the factors. The $\operatorname{set}^{\mathrm{FACT}_{n}}(c)$ is also called the set of reduced decompositions of $c$ into reflections.

If $\left(w_{1}, \ldots, w_{p}\right)$ is a factorization of $c$, then we get a (strict) chain in $\mathrm{NC}(W)$ :

$$
w_{1} \prec w_{1} w_{2} \prec \cdots \prec w_{1} \ldots w_{p}=c .
$$

Strict chains are related to multichains by straightforward formulas, so that we can pass from enumeration of multichains in $\mathrm{NC}(W)$ to enumeration of block factorizations of $c$, and vice versa (see [Rip10a. App. B] or [Sta97, Ch. 3.11] for example).

In the following section, we describe a geometric construction of these block factorizations, and how they are related to the fibers of a topological covering.

\footnotetext{
(vi) In contrast with the weak length $\ell_{S}$, relative to the set $S$ of Coxeter generators, which exists only in the real case.

(vii) Because all the Coxeter elements are conjugated, and the reflection length is invariant under conjugation.

(viii) We will often simply write factorization in the following.
} 


\section{Lyashko-Looijenga covering and factorizations of a Coxeter el- ement}

\subsection{Discriminant of a well-generated reflection group and Lyashko-Looijenga covering}

Let $W$ be a well-generated, irreducible complex reflection group $W$, with invariant polynomials $f_{1}, \ldots, f_{n}$, homogeneous of degrees $d_{1} \leq \cdots \leq d_{n}=h$. Note that the quotient space $V / W$ is then isomorphic to $\mathbb{C}^{n}$ :

$$
\begin{aligned}
V / W & \stackrel{\sim}{\rightarrow} \mathbb{C}^{n} \\
\bar{v} & \mapsto\left(f_{1}(v), \ldots, f_{n}(v)\right) .
\end{aligned}
$$

We recall here the construction of the Lyashko-Looijenga map of type $W$ (see [Bes07, Sec. 5] or [Rip10c, Sec. 3] for details).

We denote by $\Delta_{W}$ the discriminant of $W$. It is a polynomial in $\mathbb{C}[V]^{W}=\mathbb{C}\left[f_{1}, \ldots, f_{n}\right]$, and it is an equation of the image of the union of the reflecting hyperplanes in the quotient space $V / W$. It is known (see e.g. [Bes07, Thm. 2.4]) that when $W$ is well-generated, the fundamental invariants $f_{1}, \ldots, f_{n}$ can be chosen such that the discriminant of $W$ is a monic polynomial of degree $n$ in $f_{n}$ of the form:

$$
\Delta_{W}=f_{n}^{n}+a_{2} f_{n}^{n-2}+\cdots+a_{n},
$$

with $a_{i} \in \mathbb{C}\left[f_{1}, \ldots, f_{n-1}\right]$ (see e.g. [Bes07, Thm. 2.4]). Let us denote by $\mathcal{H}$ the discriminant hypersurface:

$$
\mathcal{H}:=\left\{\bar{v} \in V / W \mid \Delta_{W}(\bar{v})=0\right\} .
$$

and define $Y:=\operatorname{Spec} \mathbb{C}\left[f_{1}, \ldots, f_{n-1}\right] \simeq \mathbb{C}^{n-1}$, so that $V / W \simeq Y \times \mathbb{C}$.

The monic property given above implies that if we fix $f_{1}, \ldots, f_{n-1}$, then $\Delta_{W}$ always has $n$ roots (counting multiplicities) in $f_{n}$. Or, geometrically, that the intersection of the hypersurface $\mathcal{H}$ with the complex line $\left\{\left(y, f_{n}\right) \mid f_{n} \in \mathbb{C}\right\}$ (for a fixed $y \in Y$ ) generically has cardinality $n$. The definition of the Lyashko-Looijenga map comes from these observations.

Definition 5 We denote by $E_{n}$ be the set of centered configurations of $n$ points in $\mathbb{C}$, that is

$$
E_{n}:=H_{0} / \mathfrak{S}_{n}, \text { where } H_{0}=\left\{\left(x_{1}, \ldots, x_{n}\right) \in \mathbb{C}^{n} \mid \sum_{i} x_{i}=0\right\} .
$$

The Lyashko-Looijenga map of type $W$ is:

$$
\begin{aligned}
Y & \stackrel{\mathrm{LL}}{\longrightarrow} E_{n} \\
y=\left(f_{1}, \ldots, f_{n-1}\right) & \mapsto \quad \text { multiset of roots of } \Delta_{W}\left(f_{1}, \ldots, f_{n}\right) \text { in the variable } f_{n} .
\end{aligned}
$$

Remark 6 We can also see LL as an algebraic morphism. Indeed, the natural coordinates for $E_{n}$ as an algebraic variety are the $n-1$ elementary symmetric polynomials $e_{2}\left(x_{1} \ldots, x_{n}\right), \ldots, e_{n}\left(x_{1}, \ldots, x_{n}\right)$. Thus, the algebraic version of the map LL is simply the morphism

$$
\widehat{\mathrm{LL}}: \begin{array}{ccc}
\mathbb{C}^{n-1} & \rightarrow & \mathbb{C}^{n-1} \\
\left(f_{1}, \ldots, f_{n-1}\right) & \mapsto\left(a_{2}\left(f_{1}, \ldots, f_{n-1}\right), \ldots, a_{n}\left(f_{1}, \ldots, f_{n-1}\right)\right) .
\end{array}
$$




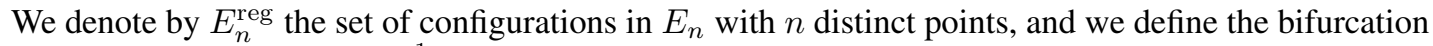
locus of LL, namely $\mathcal{K}:=\mathrm{LL}^{-1}\left(E_{n}-E_{n}^{\text {reg }}\right)$. Equivalently, we have

$$
\mathcal{K}:=\left\{y \in Y \mid D_{\mathrm{LL}}(y)=0\right\},
$$

where $D_{\mathrm{LL}}$ is called the LL-discriminant and is defined as:

$$
D_{\mathrm{LL}}:=\operatorname{Disc}\left(\Delta_{W}\left(y, f_{n}\right) ; f_{n}\right) \in \mathbb{C}\left[f_{1}, \ldots, f_{n-1}\right] .
$$

The first important property is the following (from [Bes07, Thm. 5.3]):

$$
\text { The restriction of } \mathrm{LL}: Y-\mathcal{K} \rightarrow E_{n}^{\mathrm{reg}} \text { is a topological covering of degree } \frac{n ! h^{n}}{|W|}
$$

\subsection{Geometric construction of factorizations}

\section{Discriminant stratification}

Before explaining the construction of factorizations from the discriminant hypersurface, we recall some useful properties of the geometric stratification associated to the parabolic subgroups of $W$.

The space $V$, together with the hyperplane arrangement $\mathcal{A}$, admits a natural stratification by the flats, namely, the elements of the intersection lattice $\mathcal{L}:=\left\{\bigcap_{H \in \mathcal{B}} H \mid \mathcal{B} \subseteq \mathcal{A}\right\}$.

As the $W$-action on $V$ maps a flat to a flat, this stratification gives rise to a quotient stratification $\overline{\mathcal{L}}$ of $W \backslash V$ :

$$
\overline{\mathcal{L}}=W \backslash \mathcal{L}=(p(L))_{L \in \mathcal{L}}=(W \cdot L)_{L \in \mathcal{L}},
$$

where $p$ is the projection $V \rightarrow W \backslash V$. For each stratum $\Lambda$ in $\overline{\mathcal{L}}$, we denote by $\Lambda^{0}$ the complement in $\Lambda$ of the union of the strata strictly included in $\Lambda$. The $\left(\Lambda^{0}\right)_{\Lambda \in \overline{\mathcal{L}}}$ form an open stratification of $W \backslash V$, called the discriminant stratification.

There is a natural bijection between the set of flats in $V$ and the set of parabolic subgroups of $W$ (Steinberg's theorem). This leads to a bijection between the stratification $\overline{\mathcal{L}}$ and the set of conjugacy classes of parabolic subgroups. Moreover, $\overline{\mathcal{L}}$ is in bijection with the set of conjugacy classes of parabolic Coxeter elements (which are Coxeter elements of parabolic subgroups), and with the set of conjugacy classes of elements of $\mathrm{NC}(W)$. Through these bijections, the codimension of a stratum $\Lambda$ corresponds to the rank of the associated parabolic subgroup and to the length of the parabolic Coxeter element. We refer to [Rip10c, Sect.6] for details and proofs.

\section{Geometric factorizations and compatibilities}

In [Rip10c] we established a way to exhibit factorizations from the geometry of the discriminant hypersurface $\mathcal{H}$. The starting point is the construction of a map

$$
\begin{aligned}
\rho: \mathcal{H} & \rightarrow W \\
(y, x) & \mapsto c_{y, x},
\end{aligned}
$$

by the following steps (note that $(y, x)$ lies in $\mathcal{H}$ if and only if the multiset LL $(y)$ contains $x$ ).

1. Consider a small loop in $\mathbb{C}^{n}-\mathcal{H}$, which always stays in the fiber $\{(y, t), t \in \mathbb{C}\}$, and turns once around $x$ (but not around any other $x^{\prime}$ in $\mathrm{LL}(y)$ ). 
2. This loop determines an element $b_{y, x}$ of $\pi_{1}\left(\mathbb{C}^{n}-\mathcal{H}\right)=\pi_{1}\left(V^{\text {reg }} / W\right)=B(W)$, i.e. , the braid group of $W$.

3. Send $b_{y, x}$ to $c_{y, x}$ via a fixed surjection $B(W) \rightarrow W$.

The details can be found in [Rip10c, Sect. 4]. The construction is adapted from that of Bessis in [Bes07. Sect. 6]. The map $\rho$ has the following fundamental properties.

(P1) If $\left(x_{1}, \ldots, x_{p}\right)$ is the ordered support of $\operatorname{LL}(y)$ (for the lexicographical order on $\left.\mathbb{C} \simeq \mathbb{R}^{2}\right)$, then the $p$-tuple $\left(c_{y, x_{1}}, \ldots, c_{y, x_{p}}\right)$ lies in $\operatorname{FACT}_{p}(c)$.

(P2) For all $x \in \mathrm{LL}(y), c_{y, x}$ is a parabolic Coxeter element; its length is equal to the multiplicity of $x$ in $\operatorname{LL}(y)$, and its conjugacy class corresponds (via the bijection mentioned above) to the unique stratum $\Lambda$ in $\overline{\mathcal{L}}$ such that $(y, x) \in \Lambda^{0}$.

According to property (P1), we call the tuple $\left(c_{y, x_{1}}, \ldots, c_{y, x_{p}}\right)$ (where $\left(x_{1}, \ldots, x_{p}\right)$ is the ordered support of LL $(y)$ ) the factorization of c associated to $y$, and we denote it by facto $(y)$.

Any block factorization determines a composition of $n$. To any configuration of $E_{n}$ we can also associate a composition of $n$, formed by the multiplicities of its elements in the lexicographical order. Then property (P2) implies that for any $y$ in $Y$, the compositions associated to $\operatorname{LL}(y)$ and facto $(y)$ are the same. The third fundamental property (see [Rip10c, Thm. 5.1] or [Bes07, Thm. 7.9]) is the following.

(P3) The map LL $\times$ facto $: Y \rightarrow E_{n} \times \operatorname{FACT}(c)$ is injective, and its image is the entire set of compatible pairs (i.e. , pairs with same associated composition).

In other words, for each $y \in Y$, the fiber $\operatorname{LL}^{-1}(\operatorname{LL}(y))$ is in bijection (via facto) with the set of factorizations whose associated composition of $n$ is the same as that associated to facto $(y)$.

\section{Combinatorics of the submaximal factorizations}

Property (P3) is particularly helpful to compute algebraically certain classes of factorizations. For example, if $y$ lies in $Y-\mathcal{K}$, then facto $(y)$ is in $\operatorname{FACT}_{n}(c)$ (in other words, it is a reduced decomposition of $c$ ), i.e. the associated composition is $(1,1, \ldots, 1)$. Thus, from (P3), the set of reduced decomposition of $c$ is in bijection with any generic fiber of LL (the fiber of any point in $E_{n}^{\text {reg }}$ ), so it has cardinality $n ! h^{n} /|W|$, because of property $(\mathrm{P} 0)$. Note that this number has been computed algebraically, using the fact that the algebraic morphism LL is "weighted-homogeneous".

The natural question is: can we go further, and obtain enumeration of more complicated factorizations, using the property (P3) and a geometric study of the morphism $\widehat{\mathrm{LL}}$ ? This section gives a positive answer for the case of submaximal factorizations.

\subsection{Restriction of LL and submaximal factorizations of a given type}

A submaximal factorization of a Coxeter element $c$ is a block factorization of $c$ whose underlying partition of the associated composition is $\alpha=2^{1} 1^{n-2} \vdash n$. In other words, these are factorizations of $c$ in $n-1$ blocks $((n-2)$ reflections and one factor of length 2$)$, and as such, they are a natural first generalization of the set of reduced decompositions $\mathrm{FACT}_{n}(c)$. 
Obviously we have to study the restriction of LL to the bifurcation locus $\mathcal{K}$. In fact, it is easier to first study finer restrictions, because $\mathcal{K}$ is not irreducible. Before stating the properties that we obtain, we need some notations.

Definition 7 We call $\overline{\mathcal{L}}_{2}$ the set of strata of $\overline{\mathcal{L}}$ of codimension 2. Its elements correspond (via the bijection described earlier) to the conjugacy classes of parabolic Coxeter elements of length 2.

We define $\varphi$ to be the projection:

$$
\begin{aligned}
V / W \simeq Y \times \mathbb{C} & \stackrel{\varphi}{\rightarrow} Y \\
(y, x) & \mapsto y .
\end{aligned}
$$

Let $\Lambda$ be a stratum of $\overline{\mathcal{L}}_{2}$. We define:

- $\operatorname{FACT}_{n-1}^{\Lambda}(c)$ : the set of submaximal factorizations of type $\Lambda$, i.e. factorizations whose unique factor of length 2 has a conjugacy class corresponding to $\Lambda$.

- $E_{\alpha}:=E_{n}-E_{n}^{\mathrm{reg}}$.

- $E_{\alpha}^{0}$ : the subset of $E_{\alpha}$ consisting of configurations whose partition of multiplicities is $\alpha=2^{1} 1^{n-2} \vdash$ $n$.

- $\varphi(\Lambda)^{0}:=\varphi(\Lambda) \cap \mathrm{LL}^{-1}\left(E_{\alpha}^{0}\right)$.

- $\mathcal{K}^{0}=\mathrm{LL}^{-1}\left(E_{\alpha}^{0}\right)=\bigcup_{\Lambda \in \overline{\mathcal{L}}_{2}} \varphi(\Lambda)^{0}$.

- $\mathrm{LL}_{\Lambda}$ : the restriction of $\mathrm{LL}: \varphi(\Lambda) \stackrel{\mathrm{LL}_{\Lambda}}{\longrightarrow} E_{\alpha}$.

Then, from [Rip10c], we have the following properties.

(i) The irreducible components of $\mathcal{K}$ are the $\varphi(\Lambda)$, for $\Lambda \in \overline{\mathcal{L}}_{2}$.

(ii) The connected components of $\mathcal{K}^{0}$ are the $\varphi(\Lambda)^{0}$, for $\Lambda \in \overline{\mathcal{L}}_{2}$.

(iii) The restriction of LL : $\mathcal{K}^{0} \rightarrow E_{\alpha}^{0}$ is a (possibly not connected) unramified covering [Rip10c, Thm. 5.2].

(iv) The image, by the map facto, of $\varphi(\Lambda)^{0}$ is exactly FACT ${ }_{n-1}^{\Lambda}(c)$.

For each $\Lambda$, let us denote by $D_{\Lambda}$ an irreducible polynomial in $f_{1}, \ldots, f_{n-1}$ such that $\varphi(\Lambda)=\left\{D_{\Lambda}=0\right\}$. From (i) we obtain a decomposition of the polynomial $D_{\mathrm{LL}}$ (the equation of $\mathcal{K}$, see Sec. 3.1):

$$
D_{\mathrm{LL}}=\prod_{\Lambda \in \overline{\mathcal{L}}_{2}} D_{\Lambda}^{r_{\Lambda}}, \text { for some } r_{\Lambda} \geq 1
$$

Using the algebraic property of the restriction $\mathrm{LL}_{\Lambda}$ defined above, we can then obtain the following results.

Theorem 8 Let $\Lambda$ be a strata of $\overline{\mathcal{L}}_{2}$. Then: 
(a) $\mathrm{LL}_{\Lambda}$ is a finite quasi-homogeneous morphism of degree $\frac{(n-2) ! h^{n-1}}{|W|} \operatorname{deg} D_{\Lambda}$;

(b) the number of submaximal factorizations of cof type $\Lambda$ is equal to

$$
\left|\operatorname{FACT}_{n-1}^{\Lambda}(c)\right|=\frac{(n-1) ! h^{n-1}}{|W|} \operatorname{deg} D_{\Lambda} .
$$

Proof: (outline) We use the fact that the map $\mathrm{LL}_{\Lambda}$ defined above can be viewed as an algebraic morphism $\widehat{\mathrm{LL}}_{\Lambda}$, corresponding to the extension

$$
\mathbb{C}\left[a_{2}, \ldots, a_{n}\right] /(D) \subseteq \mathbb{C}\left[f_{1}, \ldots, f_{n-1}\right] /\left(D_{\Lambda}\right)
$$

Then we compute Hilbert series to obtain the degree of this extension.

(b) is a direct consequence of (a) together with Property (P3). The multiplicative factor $(n-1)$ is because there are $(n-1)$ compositions of $n$ whose underlying partition is $2^{1} 1^{n-2}$.

Remark 9 In [KM10], motivated by the enumerative theory of the generalized non-crossing partitions, Krattenthaler and Müller defined and computed the decomposition numbers of a Coxeter element, for all irreducible real reflection groups. In our terminology, these are the numbers of block factorizations according to the Coxeter type of the factors. Note that the Coxeter type of a parabolic Coxeter element is the type of its associated parabolic subgroup, in the sense of the classification of finite Coxeter groups. So the conjugacy class for a parabolic elements is a finer characteristic than the Coxeter type (ix)

Nevertheless, when $W$ is rea ${ }^{(\mathrm{x})}$. most of the results obtained from formula $(b)$ in Thm. 8 are very specific cases of the computations in [KM10]. But the method of proof is completely different, geometric instead of combinatorial. Note that another possible way to tackle this problem is to use a recursion, to obtain data for the group from the data for its parabolic subgroups. A recursion formula (for factorizations where the rank of each factor is dictated) is indeed given by Reading in [Rea08], but the proof is very specific to the real case.

For $W$ non-real, formula (b) implies new combinatorial results on the factorizations of a Coxeter element. The numerical data for all irreducible well-generated complex reflection groups are gathered in the Table 2 of [Rip10b]. In particular, we obtain (geometrically) general formulas for the submaximal factorizations of a given type in $G(e, e, n)$.

\subsection{Jacobian and discriminant of $\mathrm{LL}$}

Now we would like to compute the total number of submaximal factorizations. For this, we need to work out the sum of the formulas of Thm. 8(b). The problem is that we don't know explicitly the degrees of the polynomials $D_{\Lambda}$.

We recall that $D_{\mathrm{LL}}=\prod_{\Lambda \in \overline{\mathcal{L}}_{2}} D_{\Lambda}^{r_{\Lambda}}$. We use the following theorem to get through it in a uniform way.

(ix) Take for example $D_{4}$, where there are three conjugacy classes of parabolic elements of type $A_{1} \times A_{1}$.

(x) The computation of all decomposition numbers for complex groups, by combinatorial means, is also a work in progress (Krattenthaler, personal communication). 
Theorem 10 Define $J_{\mathrm{LL}}$ to be the Jacobian of the morphism $\widehat{\mathrm{LL}}$ :

$$
J_{\mathrm{LL}}=\operatorname{Jac}\left(\left(a_{2}, \ldots, a_{n}\right) /\left(f_{1}, \ldots, f_{n-1}\right)=\operatorname{det}\left(\frac{\partial a_{i}}{\partial f_{j}}\right)_{\substack{1 \leq j \leq n-1 \\ 1 \leq j \leq n}} .\right.
$$

Then (up to a multiplicative constant):

$$
J_{\mathrm{LL}}=\prod_{\Lambda \in \overline{\mathcal{L}}_{2}} D_{\Lambda}^{r_{\Lambda}-1}
$$

The proof involves a detailed study of the polynomial ring extension associated to $\widehat{\mathrm{LL}}$. We prove that the polynomials $D_{\Lambda}$ correspond to the ramified ideals of this extension, and that the integers $r_{\Lambda}$ are their ramification indices. We also interpret them combinatorially, using the covering properties of LL. Then we deduce that the extension is "well-ramified", as defined in [Rip10a. Ch. 2], which implies the expected factorization of the Jacobian. We refer to [Rip10b, Thm. 3.4] for details.

\subsection{Enumeration of submaximal factorizations}

We deduce easily the computation of the total number of submaximal factorizations.

Corollary 11 Let $W$ be an irreducible, well-generated complex reflection group, with invariant degrees $d_{1} \leq \cdots \leq d_{n}=h$. Then, the number of submaximal factorizations of a Coxeter element $c$ is equal to:

$$
\left|\operatorname{FACT}_{n-1}(c)\right|=\frac{(n-1) ! h^{n-1}}{|W|}\left(\frac{(n-1)(n-2)}{2} h+\sum_{i=1}^{n-1} d_{i}\right) .
$$

Proof: Using Thm. 8(b) and Thm. 10, we obtain:

$$
\begin{aligned}
& \left|\operatorname{FACT}_{n-1}(c)\right|=\sum_{\Lambda \in \overline{\mathcal{L}}_{2}}\left|\mathrm{FACT}_{n-1}^{\Lambda}(c)\right| \\
& =\frac{(n-1) ! h^{n-1}}{|W|} \sum_{\Lambda \in \overline{\mathcal{L}}_{2}} \operatorname{deg} D_{\Lambda} \\
& =\frac{(n-1) ! h^{n-1}}{|W|}\left(\operatorname{deg} D_{\mathrm{LL}}-\operatorname{deg} J_{\mathrm{LL}}\right) \text {, }
\end{aligned}
$$

As $D_{\mathrm{LL}}$ is a classical discriminant with respect to the variable $f_{n}$ of degree $h$, we have $\operatorname{deg} D_{\mathrm{LL}}=$ $n(n-1) h$. Moreover: $\operatorname{deg} J_{\mathrm{LL}}=\sum_{i=2}^{n} \operatorname{deg}\left(a_{i}\right)-\sum_{j=1}^{n-1} \operatorname{deg}\left(f_{j}\right)=\sum_{i=2}^{n} i h-\sum_{j=1}^{n-1} d_{j}$. A quick computation allows to conclude.

The formula in the above theorem is actually included in Chapoton's formula: indeed, there exist easy combinatorial tricks allowing to pass from the numbers of multichains to the numbers of strict chains, which are roughly the numbers of block factorizations (see [Rip10a, App. B] for details.

However, the proof we obtained here is more satisfactory (and more enlightening) than the one using Chapoton's formula. Indeed, if we recapitulate the ingredients of the proof, we only made use of the formula for the number of reduced decompositions - necessary to prove the first properties of LL in 
[Bes07]-, the remaining being the geometric properties of LL, for which we never used the classification. In other words, we travelled from the numerology of $\mathrm{FACT}_{n}(c)$ to that of $\mathrm{FACT}_{n-1}(c)$, without adding any case-by-case analysis to the setting of [Bes07].

Yet, the method used here to compute the number of submaximal factorizations is difficult to generalize to factorizations with fewer blocks. A more promising approach would be to avoid computing explicitely these factorizations, and to try to understand globally Chapoton's formula as some ramification formula for the morphism LL. A reformulation of the formula gives indeed:

$$
\forall p \in \mathbb{N}, \sum_{k=1}^{n}\left(\begin{array}{c}
p+1 \\
k
\end{array}\right)\left|\operatorname{FACT}_{k}(c)\right|=\prod_{i=1}^{n} \frac{d_{i}+p h}{d_{i}},
$$

where the $\left|\operatorname{FACT}_{k}(c)\right|$ are closely related to the cardinalities of the fibers of LL.

\section{Acknowledgements}

Most of this work has been carried out during my PhD thesis. I would like to thank heartily my advisor David Bessis for his constant support and his help on many points. I am also grateful to Raphaël Rouquier for suggesting to me an essential point in the proof of Thm. 10

\section{References}

[AR04] Christos A. Athanasiadis and Victor Reiner. Noncrossing partitions for the group $D_{n}$. SIAM J. Discrete Math., 18(2):397-417 (electronic), 2004.

[Arm09] Drew Armstrong. Generalized noncrossing partitions and combinatorics of Coxeter groups. Mem. Amer. Math. Soc., 202(949):x+159, 2009.

[BC06] David Bessis and Ruth Corran. Non-crossing partitions of type $(e, e, r)$. Adv. Math., 202(1):149, 2006.

[Bes07] David Bessis. Finite complex reflection arrangements are $K(\pi, 1)$. Preprint arXiv:math/0610777v3, 2007.

[Cha05] Frédéric Chapoton. Enumerative properties of generalized associahedra. Sém. Lothar. Combin., 51:Art. B51b, 16 pp. (electronic), 2004/05.

[KM10] Christian Krattenthaler and Thomas W. Müller. Decomposition numbers for finite Coxeter groups and generalised non-crossing partitions. Trans. Amer. Math. Soc., 362:2732-2787, 2010.

[Kre72] Germain Kreweras. Sur les partitions non croisées d'un cycle. Discrete Mathematics, 1(4):333$350,1972$.

[LT09] Gustav I. Lehrer and Donald E. Taylor. Unitary reflection groups, volume 20 of Australian Mathematical Society Lecture Series. Cambridge University Press, Cambridge, 2009.

[Rea08] Nathan Reading. Chains in the noncrossing partition lattice. SIAM J. Discrete Math., 22(3):875886, 2008. 
[Rei97] Victor Reiner. Non-crossing partitions for classical reflection groups. Discrete Math., 177(13):195-222, 1997.

[Rip10a] Vivien Ripoll. Groupes de réflexion, géométrie du discriminant et partitions non-croisées. $\mathrm{PhD}$ thesis, Université Paris Diderot-Paris 7, 2010. arXiv:1010.4349.

[Rip10b] Vivien Ripoll. Lyashko-Looijenga morphisms and submaximal factorisations of a Coxeter element. Preprint arXiv:1012.3825, December 2010.

[Rip10c] Vivien Ripoll. Orbites d'Hurwitz des factorisations primitives d'un élément de Coxeter. J. Alg., 323(5), March 2010.

[ST54] Geoffrey C. Shephard and John A. Todd. Finite unitary reflection groups. Canadian J. Math., 6:274-304, 1954.

[Sta97] Richard P. Stanley. Enumerative combinatorics. Vol. 1, volume 49 of Cambridge Studies in Advanced Mathematics. Cambridge University Press, Cambridge, 1997. With a foreword by Gian-Carlo Rota, Corrected reprint of the 1986 original. 